\title{
A generalisation of the nine-point circle and Euler line
}

\author{
Michael de Villiers
}

University of KwaZulu-Natal

Email: profmd@mweb.co.za

"Now I will have less distraction." Leonhard Euler, referring to losing the sight of one eye, quoted in Mathematical Circles (Howard Eves, 1969)

\section{Introduction}

To most people, including some mathematics teachers, geometry is synonymous with ancient Greek geometry, especially as epitomised in Euclid's Elements of 300 BC. Sadly, many are not even aware of the significant extensions and investigations of Apollonius, Ptolemy, Pappus, and many others until about 320 AD. Even more people are completely unaware of the major developments that took place in synthetic Euclidean plane geometry from about 1750-1940, and more recently again from about 1990 onwards (stimulated in no small way by the current availability of dynamic geometry software).

The purpose of this article is therefore to give a brief historical background to the discovery of the Nine-point circle and the Euler line, and a simple, but possibly new generalisation and proof of the latter, that may be of interest to teachers and students.

\section{High school background}

The reader is reminded of the following three classic concurrency results from Euclid's Elements that are fortunately still mentioned (though seldom with proof) in a few South African high school textbooks. However, since these results are no longer required "theorems" for the final matricu-

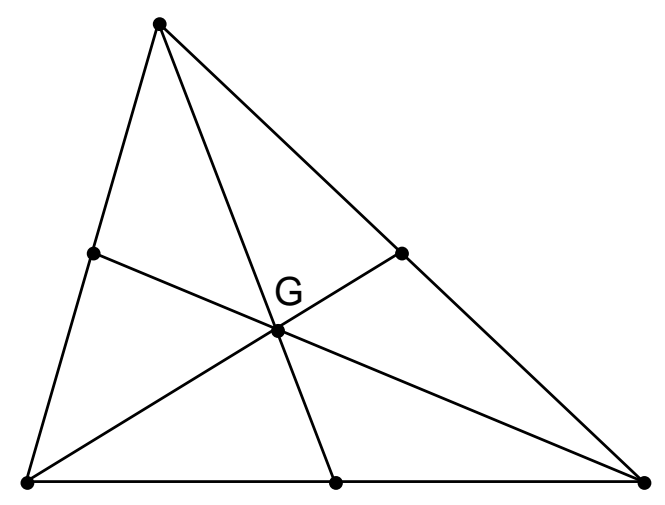

Figure 1. Centroid lation examination from about the late 1980s, it is likely that most teachers have simply ignored teaching them, thus producing a generation of children unacquainted with these remarkable

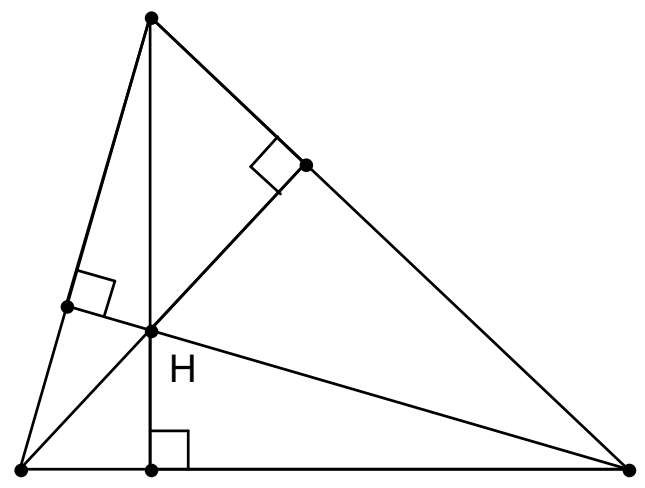

Figure 2. Orthocentre

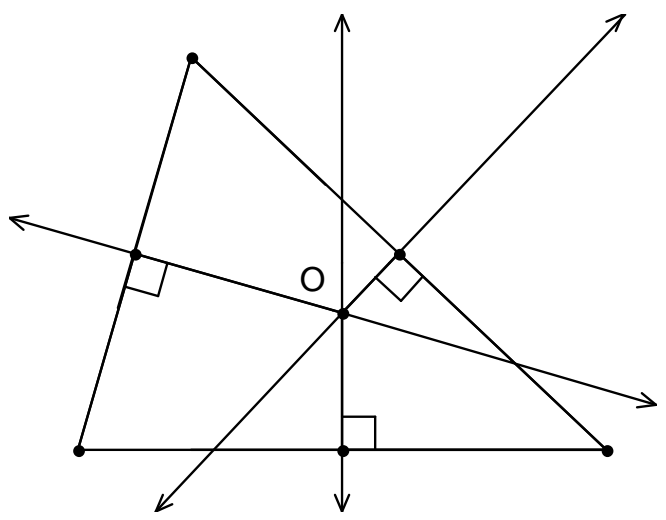

Figure 3. Circumcentre

results from our geometric heritage.

(1) The medians (lines from the vertices to the midpoints of the opposite sides) of a triangle are concurrent at the centroid (centre of gravity) of a triangle (see Figure 1).

(2) The altitudes (perpendicular lines from the vertices to the opposite sides) of a triangle are concurrent at the orthocentre (see Figure 2).

(3) The perpendicular bisectors of the sides (lines through the midpoints of the sides and perpendicular to them) of a triangle are concurrent at the circumcentre, which is the centre of the circle through the three vertices (see Figure 3). 


\section{Ceva's theorem}

In 1678 an Italian mathematician named Giovanni Ceva discovered a surprising generalisation of the altitude and median (and angle bisector) concurrencies, namely, that if in any triangle, line segments $A D, B F$ and $C E$ are concurrent (with $D$, $F$ and $E$ respectively on sides $B C, A C$ and $A B$ ), then $\frac{A F}{F C} \times \frac{C D}{D B} \times \frac{B E}{E A}=1 . \quad$ Conversely, if $\frac{A F}{F C} \times \frac{C D}{D B} \times \frac{B E}{E A}=1$, then line segments $A D, B F$ and $C E$ are concurrent (see Figure 4). In Ceva's honour, the line segments $A D, B F$ and $C E$ joining the vertices of a triangle to any given points on the opposite sides, are called cevians.

Ceva's theorem is a very important and useful theorem that has to form part of the standard armoury of any high school learner aspiring to be competitive at the Third Round level of the South African Mathematics Olympiad. Learners who participate in the workshops and Summer School of the Mathematical Talent Search organised under the auspices of the South African Mathematical Society (SAMS) are well acquainted with this result, as are all the South African team members of the International Mathematics Olympiad (IMO).

\section{Homothetic polygons}

Another valuable result that is usually also well known to successful Mathematics Olympiad contestants is the following theorem: If two polygons are homothetic (that is similar and their corresponding sides are parallel), then the lines connecting corresponding vertices are concurrent at their centre of similarity (see Figure 5).
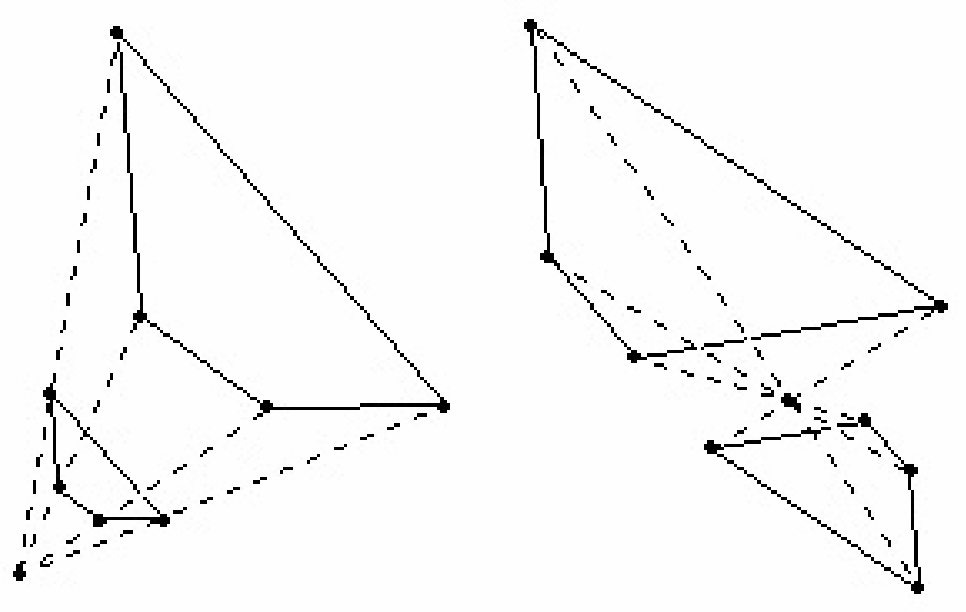

Figure 5. Homothetic polygons

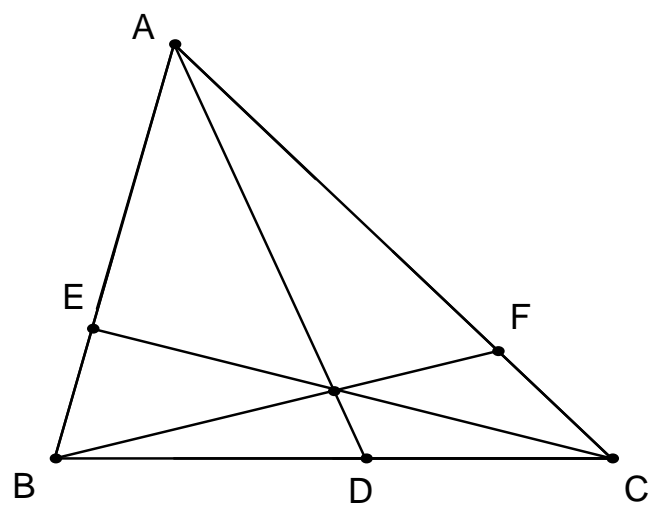

Figure 4. Ceva's theorem

\section{The nine-point circle and Euler line}

Although Leonhard Euler was apparently the first person in 1765 to show that the midpoints of the sides of a triangle and the feet of the altitudes determine a unique circle, it was not until 1820 that Brianchon and Poncelet showed that the three midpoints of the segments from the orthocentre to the vertices also lie on the same circle, hence its name, the nine-point circle (see Figure 6). The nine-point circle is often also referred to as the Euler circle in honour of Euler. It is also sometimes called the Feuerbach circle in honour of Karl Feuerbach who in 1822 proved the stunning theorem that the nine-point circle is tangent to the incircles and excircles of the triangle!

A result closely associated with the nine-point circle is that of the Euler line (which Euler presumably discovered more or less simultaneously), namely that the orthocentre $(H)$, centroid $(G)$, circumcentre $(O)$ and the centre of nine-point circle $(N)$ are collinear. Moreover, $H G=2 G O$ and $H N=$ $3 N G$.

The historical background referred to above is widely available in standard historical resource books like Boyer (1968), Kramer (1970), etc. Books such as these ought to be regularly consulted by teachers and lecturers in order to bring a much-needed historical perspective to mathematics in the classroom. For classic synthetic proofs of the results mentioned above, readers can for example consult any advanced geometry textbook like Coxeter and Greitzer (1967) or Posamentier (2002). 


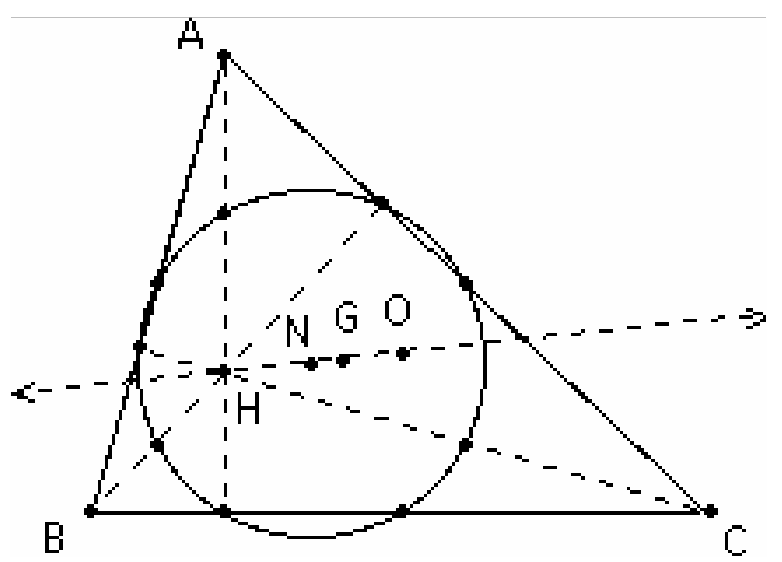

Figure 6. Nine-point circle \& Euler line

\section{Experimental discovery}

In October 2002, I was wondering how one might generalise the nine-point circle and started off by considering what happens if instead of the concurrent altitudes, one took any three concurrent cevians (lines from the vertices to the opposite sides). Next I constructed the midpoints of the segments from the cevian point $H$ to the vertices as shown in Figure 7, wondering whether there was any significance in them. Dynamically dragging and manipulating the triangle with Sketchpad for a while, it suddenly visually seemed to suggest that the feet of the cevians $D, E$ and $F$, and the midpoints $J, K$ and $L$ all lie on an ellipse. This was immediately confirmed when I used a Sketchpad tool to draw an ellipse through any five of these points, the ellipse passing through the remaining sixth point. Much to my surprise, and delight, I next noticed with further dragging that this ellipse always passed through the midpoints of the sides of triangle $A B C$ (and turned into a hyperbola if $D$, $E$ and $F$ was dragged onto the extensions of the sides of the triangle). In other words, nine points in total lie on this uniquely determined conic!

After labouring through long analytic geometry proofs (with the aid of the symbolic computer algebra of the TI-92), I later found out, much to my dismay, that the discovery was not novel at all, and was already known in the $1890 \mathrm{~s}$, appearing in some projective geometry texts (Russell, 1893: 212). It also appears in standard projective geometry texts such as Baker (1922: 41-42), which were required study material for entrance examinations into the mathematical doctoral programmes of Oxford and Cambridge in the 1920 s and 1930s. It seems quite sad that such a beautiful projective geometry result has become forgotten and neglected.
This nine-point conic result, however, contains a generalisation of the Euler line as a corollary, which does not appear in any of the three references mentioned, and an internet search has also provided no explicit mention of it in the mathematical literature. Furthermore, my initial analytic proofs of the nine-point conic and the Euler generalisation (see De Villiers, in press), although confirming the results, do not provide any satisfactory insight into why the results are true. In contrast, the proof given further down for the Euler generalisation is not only synthetic, but also explanatory in terms of similarity.

For the sake of completeness, the nine-point conic theorem and Euler line generalisation are now formally stated, and a proof of the latter given.

\section{The nine-point conic}

Given any triangle $A B C$, and three cevians concurrent in $H$, then the feet of the cevians $(D, E$ and $F$ ), the midpoints of the sides of the triangle ( $X, Y$ and $Z$ ), and the respective midpoints $L, J$ and $K$ of the segments $H A, H B$ and $H C$, lie on a conic (Figure 7).

(Note that an ellipse is obtained when the feet of all the cevians are on the sides of the triangle, but when some of the feet of the cevians lie on the extensions of the sides, the conic becomes a hyperbola. Silvester (2001: 214-215) also explains how a six-point parabola can (theoretically) be obtained as a limiting case as the cevian point $H$ is dragged off towards infinity.)

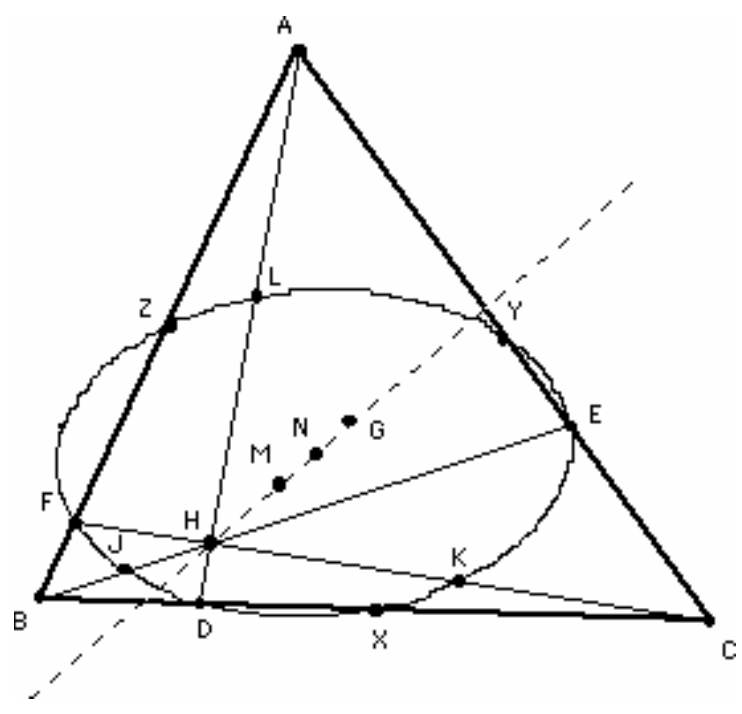

Figure 7. Nine-point conic and Euler line generalisation 


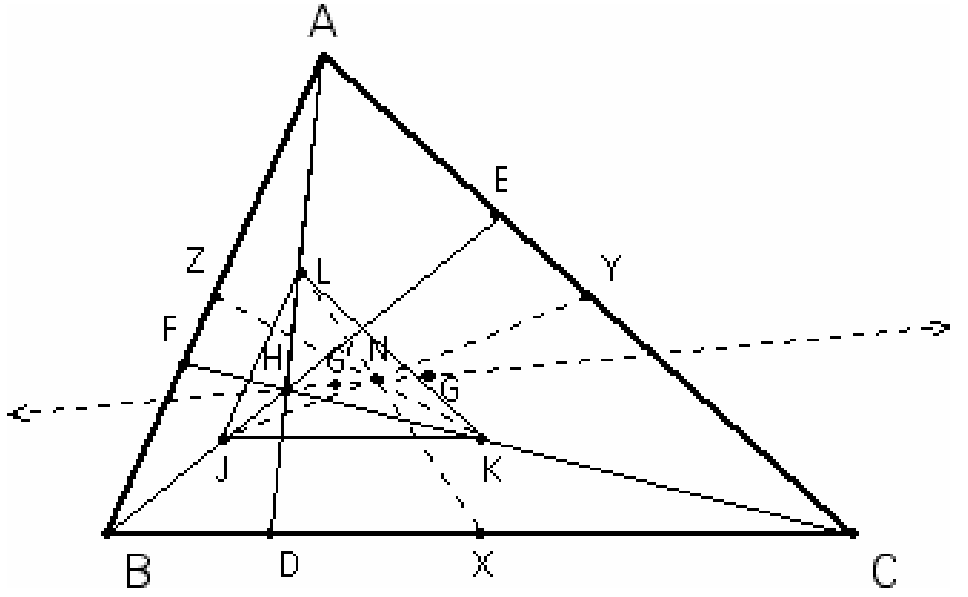

Figure 8. Further Euler Line generalisation
Further Euler line generalisation The above Euler line generalisation generalises even further as follows. Given any triangle $A B C$ with midpoints of the sides $X, Y$ and $Z$ and three cevians concurrent in $H$ as shown in Figure 8. With $H$ as centre of similarity and scale factor $\frac{1}{k}$, construct triangle $L J K$ similar to $A B C$. Let $N$ be the centre of similarity between $L J K$ and the median triangle $X Y Z$. Then $H, N$ and $G$ are collinear, and $H N=\frac{3}{k-1} N G$.

\section{Proof}

Construct the centroid $G^{\prime}$ of triangle $L J K$. Since $A B C$ maps onto $L J K$ under the similarity situated at $H$, it follows that $\frac{H G}{H G^{\prime}}=k$ and $H, G^{\prime}$ and $G$ are collinear. Since the median triangle is also similar to $A B C$ under a half-turn around $G$ with a scale factor of $\frac{1}{2}$, it follows that $L J K$ is similar to $X Y Z$ with a scale factor $\frac{2}{k}$. Moreover, $L J K$ is homothetic to $X Y Z$. Therefore lines $L X, J Y$ and $K Z$ are concurrent at $N$ (with $2 X N=k N L$, etc.). Then since the centroid of the median triangle coincides with the centroid $G$ of $A B C$, and $X Y Z$ maps onto $L J K$ under the half-turn and similarity situated at $N$, it follows that $\frac{N G}{N G}=\frac{k}{2}$ and $G^{\prime}, N$ and $G$ are collinear. Since the straight line through $G$ and $G^{\prime}$ is unique, it follows that $H, G^{\prime}, N$ and $G$ are collinear.

Let $H G^{\prime}=x$, then from the ratios into which HG is divided as shown in Figure 9, it follows that $\frac{H N}{N G}=\frac{3 x k}{k+2} \times \frac{k+2}{k x(k-1)}=\frac{3}{k-1}$

\section{Looking back}

Instead of respectively using parallelograms and homothetic polygons to prove $X L, Y J$ and $Z K$ concurrent at $N$ for the Euler line generalisation and further Euler line generalisation, Ceva's theorem could be used. further note that both Euler generalisations can be viewed as theorems about quadrilateral

Figure 9. Ratios between segments 
$A B C H$. An interesting physical interpretation of both generalisations is to consider finding the centroid $N$ of point masses at $A, B, C$ and $H$. For example, for the first Euler line generalisation, consider unit masses at $A, B, C$ and $H$. Then $N$ is the centroid of 2 masses at $X$ and $L$, etc., and also the centroid of 3 masses at $G$ and 1 at $H$; hence $H N$ $=3 N G$. Similarly, for the further Euler generalisation, consider unit masses at $A, B$ and $C$ and mass $k-1$ at $H$.

Since the further Euler generalisation no longer involves a conic, it is perhaps less interesting than the Euler generalisation, which it generalises. Indeed, this is often the case with generalisation, since the general case frequently involves fewer properties than the special case.

\section{Concluding comment}

As mathematicians we have an educational obligation to share new developments in our discipline with our learners to combat the pervasive misconception that mathematics is a sterile and dead subject. Euclidean plane geometry is particularly suited as learners can easily be led to some visual appreciation even without formal proof. Moreover, it may just stimulate their own creativity and inspire them to engage in some mathematical research themselves. The availability of dynamic geometry software also encourages a kind of experimental approach in which it is easy to make and check conjectures that lie well within the means of average learners, and not just a select few.

\section{Acknowledgements}

This article is dedicated to the recently deceased Martyn Cundy, co-author of the famous geometry classic Mathematical Models (published in 1951), who kindly suggested to me the elegant proof of the Euler line generalisation, and which led to the further Euler line generalisation.

This research was partially funded by a National Research Foundation (NRF) grant from the Spatial Orientation \& Spatial Insight (SOSI) Project, Pretoria, GUN No. 2050502.

Note: A Dynamic Geometry (Sketchpad 4) sketch in zipped format (Winzip) of the results discussed here can be downloaded directly from: http://my site.mweb.co.za/residents/profmd/9pointeuler.zip (This sketch can also be viewed with a free demo version of Sketchpad 4 that can be downloaded from: http://www.keypress.com/sketchpad/sketch demo.html)

\section{References}

Baker, H.F. (1922). Principles of Geometry, Volume II. Cambridge: Cambridge University Press.

Boyer, C.B. (1968). A History of Mathematics. New York: John Wiley \& Sons.

Coxeter, H.S.M. \& Greitzer, S.L. (1967). Geometry Revisited. Washington, DC: The Mathematical Association of America.

De Villiers, M.D. (in press). The nine-point ellipse: a rediscovery and proof by computer, The International Journal for Mathematical Education in Science \& Technology.

Eves, H. (Ed.) (1969). Mathematical Circles. Boston: Prindle, Weber \& Schmidt.

Kramer, E.E. (1970). The Nature and Growth of Modern Mathematics. Princeton: Princeton University Press.

Posamentier, A. (2002). Advanced Euclidean Geometry. Emeryville: Key College Publishing.

Russell, J. W. (1893). Pure Geometry. Oxford: Oxford University Press.

Silvester, J.R. (2001). Geometry: Ancient \& Modern. Oxford: Oxford University Press.

"Life without geometry is pointless ..."

(Author unknown) 